\title{
EQUIVALENCE OF VARIATIONAL INEQUALITIES WITH WIENER-HOPF EQUATIONS
}

\author{
PETER SHI
}

(Communicated by Barbara L. Keyfitz)

\begin{abstract}
We show that a variational inequality is equivalent to a generalized Wiener-Hopf equation in the sense that, if one of them has a solution so does the other one. Moreover, their solutions can be transformed to each other by a simple formula. Applications are considered.
\end{abstract}

\section{INTRODUCTION}

Although variational inequalities and the Wiener-Hopf equations appear to be two different subjects, we demonstrate in this paper an equivalence between the two. This allows one to study these subjects with a unified point of view and to transplant techniques suitable for equations into inequalities. In $\S 2$, we develop the theory of this equivalence. Applications are considered in $\S 3$, where we prove the convergence of an iteration scheme, which involves only integration, to solve a class of parabolic variational inequalities with unilateral constraints.

Some of the techniques used in this paper have appeared in the literature, for example [LS], [KS], to prove the Lions-Stampacchia Theorem.

\section{MAIN THEORY}

We first introduce our notation. Let $H$ be a Hilbert space and $K$ be a closed convex subset of $H$. Let $P$ be the projection operator from $H$ onto $K$ defined by

$$
\|P u-u\| \leq\|v-u\|, \quad \forall v \in K,
$$

and let $Q=I-P$, where $I$ is the identity mapping. Assume $A$ is an arbitrary operator defined on $D(A) \subset H$ which maps $D(A)$ into $H$. For a given $f \in H$, consider the variational inequality: find $u \in D(A) \cap K$ such that

$$
(A u, v-u) \geq(f, v-u), \quad \forall v \in K,
$$

where $($,$) denotes the inner product in H$. The following equation,

$$
(A P+Q) v=f,
$$

Received by the editors November 22, 1988 and, in revised form, November 17, 1989.

1980 Mathematics Subject Classification (1985 Revision). Primary 35J85; Secondary 35A15. 
is called a generalized Wiener-Hopf equation. Here $v \in H$ is to be found. If $K$ is a closed subspace, the projection operators $P$ and $Q$ are linear operators, so (2.2) reduces to a linear equation, provided that $A$ is linear. In general, (2.2) is a nonlinear equation. We refer the reader to $[\mathrm{S}]$ and $[\mathrm{P}]$ for a treatment of generalized linear Wiener-Hopf equations where examples of different types are discussed. For general discussions of variational inequalities and their applications, we refer the reader to $[\mathrm{DL}],[\mathrm{F}],[\mathrm{KS}]$, and $[\mathrm{R}]$. The main results of this section are the following:

Theorem 2.1. The variational inequality (2.1) has a solution $u$ if and only if the Wiener-Hopf equation (2.2) has a solution $v$. Moreover, $v=u+f-A u$, and $u=P v$.

Theorem 2.2. The variational inequality (2.1) has a unique solution for each $f$ if and only if the Wiener-Hopf equation (2.2) has a unique solution for each $f$. In this case, the operator $A P+Q$ is surjective and one to one. The solution of (2.1) is given by the formula

$$
u=P(A P+Q)^{-1} f .
$$

Theorem 2.3. Suppose $A$ is a linear, one-to-one mapping from $H$ onto $H$ and $K$ is a closed convex cone with vertex at the origin. Then a necessary condition for (2.1) to have a unique solution for each $f$ is that the operator $A$ admits $a$ Wiener-Hopf factorization ${ }^{1}$; i.e.,

$$
A=A_{-} A_{+} \text {, with } A_{+} P=P, A_{-} Q=Q .
$$

We remark that $(2.2)$ provides a necessary and sufficient condition for the solvability of (2.1). That is, (2.1) has a solution if and only if $f \in \operatorname{Im}(A P+Q)$. For literature studying noncoercive variational inequalities, we refer the reader to [LS], [BGT]. However, these authors do not provide necessary and sufficient conditions.

We now prove Theorem 2.1. The following lemma on projections plays a major role in the proof. It can be found, for example, in [Z].

Lemma 2.4. Given $w \in H$ and $u \in K$, then $u=P w$ if and only if

$$
(u-w, v-u) \geq 0, \quad \forall v \in K .
$$

If $K$ is a closed convex cone with vertex at the origin, then $Q$ is the projection on $K^{*}$ and $K^{* *}=K$, where $K^{*}$ is the dual cone of $K$.

Proof of Theorem 2.1. Let $u$ be a solution of (2.1). Then (2.1) can be written as

$$
(u-[u-(A u-f)], v-u) \geq 0, \quad \forall v \in K .
$$

Hence Lemma 2.4 implies that

$$
u=P(u+f-A u) .
$$

\footnotetext{
${ }^{1}$ The details of this concept can be found in [S].
} 
Consequently,

$$
Q(u+f-A u)=u+f-A u-P(u+f-A u)=f-A u .
$$

Substituting $(2.5)$ into $(2.6)$ yields the equation

$$
(A P+Q) v=f,
$$

where $v=u+f-A u$. This proves the necessity. Conversely, let $v \in H$ be a solution of (2.7) for some $f$. Then

$$
f-A P v=Q v=v-P v,
$$

but by Lemma 2.4 ,

$$
(P v-v, w-P v) \geq 0, \quad \forall w \in K .
$$

It follows from (2.8) and (2.9) that

$$
(A P v-f, w-P v) \geq 0,
$$

namely,

$$
(A P v, w-P v) \geq(f, w-P v), \quad \forall w \in K .
$$

Hence $u=P v$ is a solution of (2.1). The relation (2.8) gives

$$
v=u+f-A u \text {. }
$$

This completes the proof of Theorem 2.1.

To prove Theorem 2.2, assume (2.1) has a unique solution $u \in K$ for a given $f$. Suppose (2.2) has two solutions $v_{1}$ and $v_{2}$. Then by Theorem 2.1, one has

$$
u=P v_{1}=P v_{2} \text {. }
$$

This leads to the equality

$$
A u+Q v_{1}=A u+Q v_{2}=f \text { or } Q v_{1}=Q v_{2} .
$$

Hence (2.11) and (2.12) imply that $v_{1}=v_{2}$.

Conversely, assume (2.2) has a unique solution $v$. By Theorem 1, for any two solutions $u_{1}, u_{2}$ of $(2.1)$, one has $u_{1}=u_{2}=P v$. The formula (2.3) follows from (2.2) and the fact that $u=P v$ in Theorem 2.1.

Under the assumptions of Theorem 2.3, the equation (2.2) is equivalent to

$$
v=\left(P+A^{-1} Q\right)^{-1} A^{-1} f .
$$

If we substitute (2.13) into (2.2), it follows that

$$
(A P+Q)\left(P+A^{-1} Q\right)^{-1} A^{-1} f=f, \quad \forall f \in H .
$$

Hence

$$
A=(A P+Q)\left(P+A^{-1} Q\right)^{-1} .
$$

Let

$$
A_{+}=\left(P+A^{-1} Q\right)^{-1}, A_{-}=A P+Q
$$


Because $Q$ is the projection onto $K^{*}, P Q=Q P=0$. Hence (2.15) defines a strong Wiener-Hopf factorization of $A$.

\section{Applications}

The equivalence of (2.1) and (2.2) allows one to identify variational inequalities with appropriate nonlinear equations. As an application of this, we develop an iteration scheme to solve parabolic variational inequalities with unilateral constraints. This is done by solving the corresponding Wiener-Hopf equations using iteration. The scheme is new, and recent numerical experiments by the author and his colleagues [PSS] have shown that it is practical. To avoid technicalities and to outline the basic idea of the method in a clear manner, we select the one-phase, one-space-dimensional Stefan problem, for example. The generalization to the case of higher space dimensions is trivial.

Assume $T>0, b>a$, and let $H=L^{2}\left(0, T ; L^{2}(a, b)\right)$. Then $H$ is a Hilbert space of function $u(x, t)$ such that the mapping $u(t, \cdot):(0, T) \rightarrow$ $L^{2}(a, b)$ is square integrable, the inner product on $H$ being given by

$$
(u, v)=\int_{0}^{T} \int_{a}^{b}(u v) d x d t .
$$

Similarly, we introduce the space $V=H^{1}\left(0, T ; H^{2}(a, b)\right)$, which is a Hilbert space of functions $u(x, t)$ such that the mapping $u(t, \cdot):(0, T) \rightarrow H^{2}(a, b)$ is an $L^{2}$ function valued in $H^{2}(a, b)$ and an $H^{1}$ function valued in $L^{2}(a, b)$. The inner product of $V$ is given by

$$
\langle u, v\rangle=\int_{0}^{T} \int_{a}^{b}\left(u v+u_{t} v_{t}+u_{x} v_{x}+u_{x x} v_{x x}\right) d x d t .
$$

We use $\|\cdot\|$, as in $\S 2$, to denote the norm of $H$ induced by the inner product. For detailed properties of the above spaces we refer the reader to [LM]. We point out, however, that $V$ is compactly imbedded in $H$; i.e., the identity mapping from $V$ into $H$ is a compact operator. Moreover, the heat operator $u_{t}-u_{x x}$ maps $V$ onto $H$.

Let $\Omega=\{(x, t) ; x \in(a, b), t \in(0, T)\}$ and $\partial_{T} \Omega=\{(x, t) ; t<T\} \cap \partial \Omega$, where $\partial \Omega$ is the topological boundary of $\Omega$. Let the closed convex subset $K$ of $H$ be defined by

$$
K=\{v \in H ; v \geq 0 \text { a.e. on } \Omega\} .
$$

In view of Theorem 1.2 in [Z], the projection $P$ from $H$ onto $K$ in this case is given by

$$
(P v)(x, t)=v^{+}(x, t)=\frac{v(x, t)+|v(x, t)|}{2}, \quad v \in H,
$$

with $Q v=v-v^{+}=v^{-} . P$ and $Q$ are nonexpansive mappings. The Stefan problem can then be formulated as following [KS], [F]: given $\psi(x, t) \in$ $C^{1}\left(\partial_{T} \Omega\right)$ and $f(x, t) \in H$, find $u \in V \cap K$ such that

$$
\left(u_{t}-u_{x x}, v-u\right) \geq(f, v-u), \quad \forall v \in K,
$$


and which satisfies the boundary condition

$$
u(x, t)=\psi(x, t) \text { uniformly up to } \partial_{T} \Omega \text {. }
$$

The existence and uniqueness of the solution to (3.1)-(3.2) are well known. For a general discussion of the Stefan problem we refer the reader to [F] and [KS].

Let $G(x, t ; \xi, \tau)$ be the Green's function ${ }^{2}$ for the heat operator $(\cdot)_{t}-(\cdot)_{x x}$ on the rectangular domain $\Omega$ and let $W$ be a function space such that

$$
V \hookrightarrow W \hookrightarrow H
$$

and the imbedding $V \hookrightarrow W$ is compact. We choose a fixed number $\alpha>0$ such that the Poincaré inequality

$$
\int_{a}^{b} v_{x}^{2} d x \geq \alpha \int_{a}^{b} v^{2} d x, \quad \forall v \in H_{0}^{1}(a, b),
$$

holds. Then the solution to (3.1) and (3.2) can be obtained via the following:

Theorem 3.1. Let $0<s<1$ and $\lambda>1 / 2 \alpha$. Construct the iteration sequence $\left\{v_{n}\right\}$ via

$$
\begin{aligned}
v_{n+1}(x, t)=(1-s) & {\left[\int_{a}^{b} G(x, t ; \xi, 0) \psi(\xi, 0) d \xi+\int_{0}^{t} G_{\xi}(x, t ; a, \tau) \psi(a, \tau) d \tau\right.} \\
- & \int_{0}^{t} G_{\xi}(x, t ; b, \tau) \psi(b, \tau) d \tau+\int_{\Omega} G(x, t ; \xi, \tau) f(\xi, \tau) d \xi d \tau \\
& \left.v^{-}(x, t)-\lambda^{-1} \int_{\Omega} G(x, t ; \xi, \tau) v^{-}(\xi, \tau) d \xi d \tau\right]+s v_{n}(x, t),
\end{aligned}
$$

with $v_{0} \in H$ being arbitrary. Then the solution $u$ to (3.1) and (3.2) is given by

$$
u=\lim _{n \rightarrow \infty}\left(v_{n}^{+}+(1-s)^{-1}\left(v_{n+1}-v_{n}\right)\right) \text {, }
$$

where the limit is taken in the strong topology of $W$.

To prove this result, we need some lemmas.

Lemma 3.2 [I, Theorem 6.4.8, Theorem 6.4.12]. Let $H$ be a Hilbert space, and let $C$ be a bounded closed convex subset of $H$. Suppose $S: C \mapsto C$ is a nonexpansive mapping. For any $s \in(0,1)$ and $v_{0} \in C$, define the iterative scheme

$$
v_{n+1}=s v_{n}+(1-s) S v_{n}, \quad n=0,1,2, \ldots
$$

Then $\left\{v_{n}\right\}$ converges weakly to a fixed point of $T$, and $\left\|v_{n+1}-v_{n}\right\|$ tends to zero as $n$ goes to $\infty$.

Lemma 3.3 [Z, p. 243]. The projection $P$ is semiweakly closed in the following sense: if $v_{n}$ is a bounded sequence that tends weakly to $v^{*} \in H$ and $P v_{n}$ tends to $w$ strongly, then $P v^{*}=w$.

\footnotetext{
${ }^{2}$ Here we mean the Green's function for the initial boundary value problem. See [W], for example.
} 
Proof of Theorem 3.1. A function $u$ satisfies (3.1) if and only if it satisfies the variational inequality

$$
\left(\lambda u_{t}-\lambda u_{x x}, v-u\right) \geq(\lambda f, v-u), \lambda>0, \quad \forall v \in K .
$$

In turn, it follows from Theorem 2.1 that $u$ satisfies (3.2)-(3.3) if and only if the parabolic Wiener-Hopf equation

$$
\lambda\left(\left(v^{+}\right)_{t}-\left(v^{+}\right)_{x x}\right)+v^{-}=\lambda f,
$$

admits a solution $v\left(=v_{\lambda}\right)$ such that $v^{+}\left(=v_{\lambda}^{+}\right)$satisfies (3.2). The integral representation for the solution of the heat equation yields

$$
\begin{aligned}
v^{+}(x, t)= & \int_{a}^{b} G(x, t ; \xi, 0) \psi(\xi, 0) d \xi+\int_{0}^{t} G_{\xi}(x, t ; a, \tau) \psi(a, \tau) d \tau \\
& -\int_{0}^{t} G_{\xi}(x, t ; b, \tau) \psi(b, \tau) d \tau+\int_{\Omega} G(x, t ; \xi, \tau) f(\xi, \tau) d \xi d \tau \\
& -\lambda^{-1} \int_{\Omega} G(x, t ; \xi, \tau) v^{-}(\xi, \tau) d \xi d \tau .
\end{aligned}
$$

Indeed, following the standard theory for partial differential equations, $v$ is a solution of (3.4) with $v^{+}$satisfying (3.2) if and only if $v$ is a solution of (3.5). We denote the first four terms in (3.5) by $F(x, t)$, which is a known function in $V$ that depends only on $\psi(x, t)$ and $f(x, t)$. Let the operator $M: H \rightarrow H$ be defined by

$$
(M v)(x, t)=\int_{\Omega} G(x, t ; \xi, \tau) v(\xi, \tau) d \xi d \tau, \quad \forall v \in H .
$$

Then (3.5) can be written as

$$
v=\left(I-\lambda^{-1} M\right) v^{-}+F .
$$

Next, we show that the operator $I-\lambda^{-1} M$ is nonexpansive. Since $M$ is linear, it is sufficient to show that

$$
\left\|I-\lambda^{-1} M\right\| \leq 1,
$$

the operator norm of $I-\lambda^{-1} M$ being less than or equal to 1 . In fact, $M$ maps $H$ into $V$ and

$$
M v=0 \text { on } \partial_{T} \Omega \text { for all } v \in H .
$$

Integration by parts gives

$$
\begin{aligned}
(M v, v) & =\int_{\Omega}(M v)\left((M v)_{t}-(M v)_{x x}\right) d x d t \\
& =\frac{1}{2} \int_{a}^{b}\left(\int_{0}^{T}(M v)_{t}^{2} d t\right) d x-\int_{0}^{T}\left(\int_{a}^{b}(M v)(M v)_{x x} d x\right) d t \\
& =\frac{1}{2} \int_{a}^{b}(M v)^{2}(x, T) d x+\int_{0}^{T}\left(\int_{a}^{b}(M v)_{x}^{2} d x\right) d t \\
& \geq \alpha \int_{0}^{T}\left(\int_{a}^{b}(M v)^{2} d x\right) d t=\alpha\|M v\|^{2}
\end{aligned}
$$


where in the last step of (3.8) we have used the Poincare inequality

$$
\int_{a}^{b} v_{x}^{2} d x \geq \alpha \int_{a}^{b} v^{2} d x, \quad \forall v \in H_{0}^{1}(a, b) .
$$

On the other hand,

$$
\left\|\left(I-\lambda^{-1} M\right) v\right\|^{2}=\|v\|^{2}-2 \lambda^{-1}(v, M v)+\lambda^{-2}\|M v\|^{2} .
$$

Hence (3.7) follows directly from (3.8)-(3.9) for $\lambda>1 / 2 \alpha$. Introduce the operator $S: H \rightarrow H$ by the relation

$$
S v=\left(I-\lambda^{-1} M\right) v^{-}+F .
$$

Since the operator $I-\lambda^{-1} M$ is linear and the projection $(\cdot)^{-}$is nonexpansive, (3.7) implies that the operator $S$ is also nonexpansive. Moreover, $S$ has a unique fixed point $v$ that solves (3.5) and $S: B \rightarrow B$, where $B$ is a ball defined by

$$
B=\left\{w \in H ;\|w-v\| \leq\left\|w-v_{0}\right\|\right\} .
$$

This can be seen from the estimates that, for any $w \in B$

$$
\|v-S w\|=\|S v-S w\| \leq\|v-w\| \leq\left\|w-v_{0}\right\| .
$$

We are now able to apply Lemma 3.2 to the iterative equation (3.6). Consider the iterative sequence

$$
v_{n+1}=s v_{n}+(1-s) S v_{n}^{-}, \quad n=0,1,2, \ldots
$$

Using (3.10), it is easy to check that $\left\{v_{n}\right\}$ is exactly the same sequence as the one that appeared in Theorem 3.1, and one has

$$
\begin{aligned}
v_{n+1} & =s v_{n}+(1-s)\left(I-\lambda^{-1} M\right) v_{n}^{-}+(1-s) F \\
& =v_{n}-(1-s) v_{n}+(1-s) v_{n}^{-}-(1-s) \lambda^{-1} M v_{n}^{-}+(1-s) F \\
& =v_{n}-(1-s)\left(v_{n}^{+}+\lambda^{-1} M v_{n}^{-}-F\right),
\end{aligned}
$$

namely,

$$
(1-s)^{-1}\left(v_{n+1}-v_{n}\right)+v_{n}^{+}=-\lambda^{-1} M v_{n}^{-}+F
$$

In what follows, we need to show only that the left-hand side of $(3.11)$ converges to $u$ in the strong topology of $W$. Suppose this is not the case and let

$$
w_{n}=(1-s)^{-1}\left(v_{n+1}-v_{n}\right)+v_{n}^{+}=-\lambda^{-1} M v_{n}^{-}+F \text {. }
$$

Then for some $\delta>0$ there exists a subsequence $\left\{w_{n_{k}}\right\}$ such that

$$
\left\|w_{n_{k}}-u\right\|_{*} \geq \delta
$$

where $\|\cdot\|_{*}$ is the norm of $W$. However, $\left\{M v_{n_{k}}^{-}\right\}$is a bounded sequence in $V$ since $\left\{v_{n}\right\}$ is contained in $B$. Since $V \hookrightarrow W$ is a compact imbedding, one concludes that there exists a subsequence of $\left\{v_{n_{k}}\right\}$, still denoted by $\left\{v_{n_{k}}\right\}$, such that

$$
M v_{n_{k}}^{-} \rightarrow v^{*} \text { strongly in } W, \text { for some } v^{*} \text { in } W \subset H
$$


Hence

$$
w_{n_{k}} \rightarrow-\lambda^{-1} v^{*}+F \text { in the strong topology of } W .
$$

Recall that $v_{n_{k}+1}-v_{n_{k}}$ tends to 0 in $H$. It then follows from (3.11) and (3.13) that

$$
v_{n_{k}}^{+} \rightarrow-\lambda^{-1} v^{*}+F \text { strongly in } H .
$$

On the other hand, Lemma 3.2 shows that $v_{n_{k}}$ tends to the unique fixed point $v$ of the operator $S$ in the weak topology of $H$. Hence Lemma 3.3 and (3.14) imply that

$$
u=v^{+}=-\lambda^{-1} v^{*}+F,
$$

which contradicts (3.12) $-(3.13)$. The proof is complete.

\section{ACKNOWLEDGMENT}

The author would like to thank Professor L. Bragg, who patiently helped the author to put the paper in this form.

\section{REFERENCES}

[BGT] C. Baioocchi, F.Gastaldi, and F. Tomarelli, Some existence results on noncoercive variational inequalities, Ann. Scuola Norm. Sup. Pisa cl. Sci. 13 (1986), 617-659.

[DL] G. Duvaut and J. L. Lions, Inequalities in mechanics and physics, Springer-Verlag, Berlin, 1976.

[F] A. Friedman, Variational principles and free boundary problems, Wiley, New York, 1982.

[I] V. I. Istratescu, Fixed point theory, D. Reidel, 1981.

[KS] D. Kinderlehrer and G. Stampacchia, An introduction to variational inequalities and their applications, Academic Press, New York, 1980.

[LM] J. L. Lions and E. Magenes, Non-homogeneous boundary value problems and applications I, Springer-Verlag, Berlin, 1972.

[LS] J. L. Lions and G. Stampacchia, Variational inequalities, Comm. Pure Appl. Math. 20 (1967), 493-519.

[PSS] A. Pitonyak, P. Shi, and M. Shillor, Numerical solutions to obstacle problems by a new iteration scheme, preprint.

[P] S. Prossdorf, Einige Klassen singularer Gleichungen, Akademie-Verlag, Berlin, 1974.

[R] J. F. Rodrigues, Obstacles problems in mathematical physics, North-Holland, Amsterdam, 1987.

[S] F-O Speck, General Wiener-Hopf factorization methods, Research Notes in Math., no. 119, Pitman Advanced Publishing Program, 1985.

[W] D. V. Widder, The heat equation, Academic Press, New York, 1975.

[Z] E. H. Zarantonello (ed.), Contributions to nonlinear functional analysis, Academic Press, New York, 1971.

Department of Mathematical Sciences, Oakland University, Oakland, Michigan 